\title{
LS-3
}

\section{Laparoscopic liver resection using the new version of ENDOEYE FLEX 3D}

\author{
Gi Hong $\mathrm{CHOI}^{*}$
}

Department of Surgery, Yonsei University College of Medicine, Seoul, Korea

Lecture: ENDOEYE 3-D (OLYMPUS, Tokyo, Japan) flexible camera system has been gradually adopted for laparoscopic liver resection because it can allow for the access to the posterior side of the liver and provide a clear discrimination of the intrahepatic vascular structures. However, it requires some learning curve to use it efficiently. The new version of ENDOEYE FLEX 3D has several advantages over the previous model. Angulation of the scope is operated by joystick, which can make it easier to intuitively understand the angulation and to hold the scope from multi-directions. The new design with the joystick angulation allows various ways to hold the scope according to surgeon's preference. In addition, it has the capability of deflecting even while in hold and renewed hold design increases working space of the scope.

In this lecture, the advantages of the new version of ENODEYE FLEX 3D will be addressed and advanced laparoscopic liver resections such as right hepatectomy, central bisectionectomy and living donor right hepatectomy using this system will be presented.

Corresponding Author: Gi Hong CHOI (choigh@yuhs.ac)

Presenter: Gi Hong CHOI (choigh@yuhs.ac) 